\title{
A novel dynamic absorber using enhanced magnetorheological elastomers for powertrain vibration control
}

\author{
Nong Zhang ${ }^{1, a}$, Nga Hoang ${ }^{2, b}$ and Haiping $\mathrm{Du}^{3, \mathrm{c}}$ \\ 1,2,3 Mechatronics and Intelligent Systems Group, Faculty of Engineering \\ University of Technology, Sydney \\ PO Box 123, Broadway, NSW 2007, Australia \\ anongz@eng.uts.edu.au, bNga.Hoang@eng.uts.edu.au , chdu@eng.uts.edu.au
}

\begin{abstract}
Keywords: dynamic absorber, vibration reduction, powertrain vibration, enhanced magnetorheological elastomer.
\end{abstract}

Abstract. This paper presents a novel Adaptive Tuned Vibration Absorber (ATVA) using the enhanced magnetorheological elastomers (MREs) for powertrain vibration reduction. The MRE material used in this application includes micro-sized iron particles enhanced by adding nano-sized magnetic powders. With the enhancement, MRE's elastic modulus significantly increases due to the MR effect. In the new ATVA, the MRE plays a role as a torsional spring whose stiffness coefficient can be varied with an external magnetic field. Additionally, this ATVA could operate in shearsqueeze mode rather than shear mode. Thus, the frequency range is much wider than that of general MREs. Such property of the enhanced MRE is an advantage for constructing a smart ATVA for powertrain vibration control because the ATVA can work effectively in a wide frequency range instead of a narrow bandwidth as a conventional dynamic absorber does. Numerical simulations of a powertrain system for the second and third gear fitted with the ATVA are used to validate its effectiveness. The obtained results show that the powertrain vibration can be greatly suppressed. Particularly, the ATVA is effective in reducing the powertrain vibration not only in case of the single harmonic excitation but also for the case of the multi-harmonic excitation. Furthermore, the simulation results can be used to optimize the ATVA's design, which will be our next work.

\section{Introduction}

MREs have been a promising material for constructing ATVAs. The vital feature of MREs is that their mechanical properties such as elastic modulus can be varied under a magnetic field. Generally, the increment in shear modulus in regular MREs under MR effect is about 50-60\% so that it seems to be insufficient to create ATVAs for systems having a wide frequency range [2-3].

There have been a number of studies about how to broaden MRE's stiffness range. Studies from [4] point out that for a magnetoactive elastomer the maximum increase in tensile and compression modulus is $200 \%$ for the former and more than $300 \%$ for the latter. Also, results, in [2] have shown that an MRE with iron by volume, $35 \%$, has largest MR effect and frequency changes are 183\%, $473 \%$ and $510 \%$ in shear, longitudinal and squeeze mode respectively. Stepanov et all [5] introduced the tangential Young's modulus for describing the no-linear behavior of a new MRE and show that the maximum increase in the modulus is up to 100 times. These results are a hint that besides increasing MR effect, the ATVAs' frequency bandwidth could be broadened if suitable operating modes such as squeeze or shear-squeeze mode are used instead of simple shear mode only. However these results come from experiment and there is no theoretical approach for ATVA modeling at all. By contrast, Zhang and Li [3] suggest a theoretical model for a new MRE and its shear modulus can be predicted. According to these authors, to fabricate this new MRE the magnetizable soft material including nano-size ferrite inside polymer gel is created first. And then, carbonyl iron particles wetted by polymer chains will be coated by the soft material. The new fabrication process makes the novel MRE increase in elastic modulus extensively. Although the model is reasonable, there is a desirable demand to develop an ATVA to verify it. And this novel ATVA will be an innovation in vibration reduction for powertrain systems. 


\section{An enhanced MR model to predict shear modulus}

According to [3], the shear modulus of conventional and nano-additive MREs due to the MR effect can be simplified by following equations:

$$
\begin{aligned}
& G_{c}=6 \phi_{p} \mu_{0} \mu_{m} H_{0}^{2} \\
& G_{n}=\frac{3}{2} \phi_{p} \mu_{0} \mu_{m} H_{0}^{2}\left(\frac{2+\phi_{n}}{1-\phi_{n}}\right)^{2}
\end{aligned}
$$

Where: $\phi_{p}$ is volume fraction of particle; $\mu_{0}=4 \pi \times 10^{-7} \mathrm{H} / \mathrm{m}, \mu_{m} \approx 1$ are the vacuum and matrix relative permeability. $\mathrm{H}_{0}$ is the magnetic field strength and $\phi_{n}$ is volume fraction of nano-particles. Totally, shear modulus of the conventional and novel MRE is determined by:

$$
G=G_{r a n}+G_{M R}
$$

Here $G_{r a n}=G_{0}\left(1+2.5 \phi+14.1 \phi^{2}\right)$. With $G_{0}$ is unfilled elastomer shear modulus, $\phi$ is the volume fraction. For conventional MREs $\phi=\phi_{p}$, for the novel MRE $\phi=\phi_{p}\left(1+\frac{\phi_{n}}{2}\right)$. $\mathrm{G}_{\mathrm{MR}}$ are given either by Eq.1 or Eq.2. for the conventional MRE and novel MRE respectively.

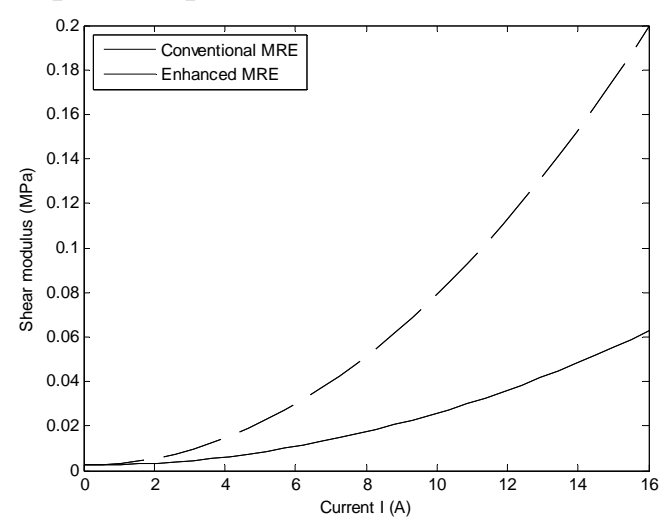

$H_{0}$ is expressed by the electromagnetic current I: $H_{0}=\alpha I$ and Eqs. 1, 2 can be re-written:

$$
\begin{aligned}
& G_{c}=6 \phi_{p} \mu_{0} \mu_{m} \alpha^{2} I^{2} \\
& G_{n}=\frac{3}{2} \phi_{p} \mu_{0} \mu_{m} \alpha^{2} I^{2}\left(\frac{2+\phi_{n}}{1-\phi_{n}}\right)^{2}
\end{aligned}
$$

For the magnetic field suggested by [7] $\alpha=12500 / \mathrm{m}$. Let $\phi_{p}=0.2, \phi_{n}=0.35, \mathrm{G}_{0}=0.001 \mathrm{MPa}$, the shear modulus of conventional and enhanced MRE calculated by Eq.3 is given in figure 1. Obviously, the enhanced MRE's shear modulus (the dashed line) is increased greatly.

Fig 1. Shear modulus of MREs

\section{A novel ATVA’s numerical simulation for powertrain vibration control}

The MRE is used to fabricate a novel ATVA whose stiffness coefficient, inertia moment $\mathrm{k}_{\mathrm{A}}, \mathrm{J}_{\mathrm{A}}$. Its natural frequency is $f_{A}=\frac{1}{2 \pi} \sqrt{\frac{k_{A}}{J_{A}}}$. If the MRE operates as a torsional spring and is modeled as a cylinder with inner, outer radius and length are a, b, L, the torsional stiffness coefficient can be, [6].

$$
k_{A}=\frac{4 \pi L a^{2} b^{2} G}{b^{2}-a^{2}}
$$

Let inertia ratio $\mu=\mathrm{J}_{\mathrm{A}} / \mathrm{J}_{3}=1 / 20\left(\mathrm{~J}_{\mathrm{A}}=0.005 \mathrm{kgm}^{2}\right), \mathrm{a}=0.05 \mathrm{~m}, \mathrm{~b}=0.06 \mathrm{~m}, \mathrm{~L}=0.012 \mathrm{~m}$. ATVA's natural frequencies depending on the input current, I, are shown in figure 2.

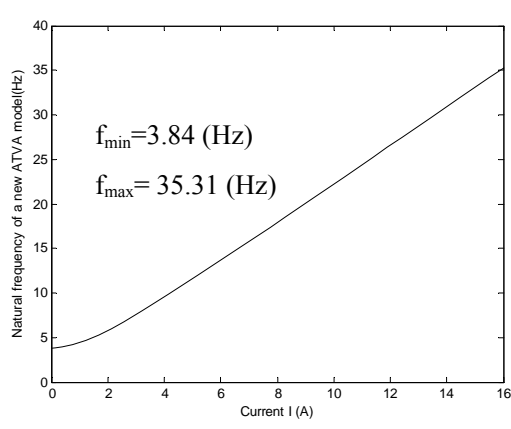

Fig 2. ATVA's frequency range

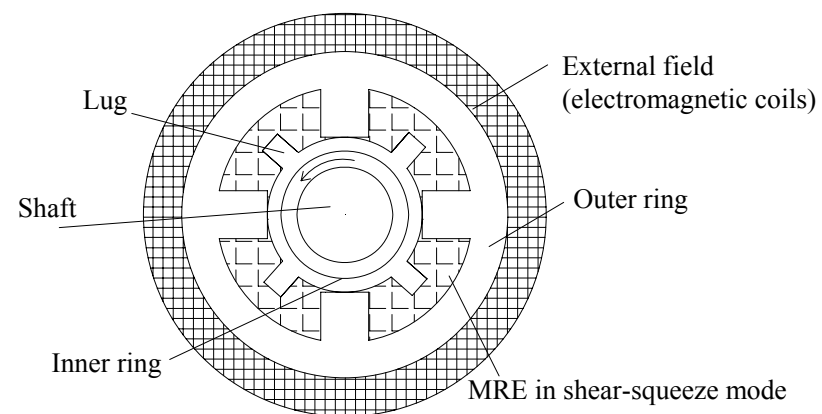

Fig 3. A proposed design for novel ATVA

If ATVA's frequency range does not cover that of powertrain, the ATVA could operate in an optimal design given in figure 3. Here the MRE is in shear-squeeze mode so that ATVA's frequency range can be broadened. Next, the ATVA's effectiveness for powertrain is discussed. 
In figure 4 a simplified powertrain model is introduced to validate the ATVA's effectiveness.

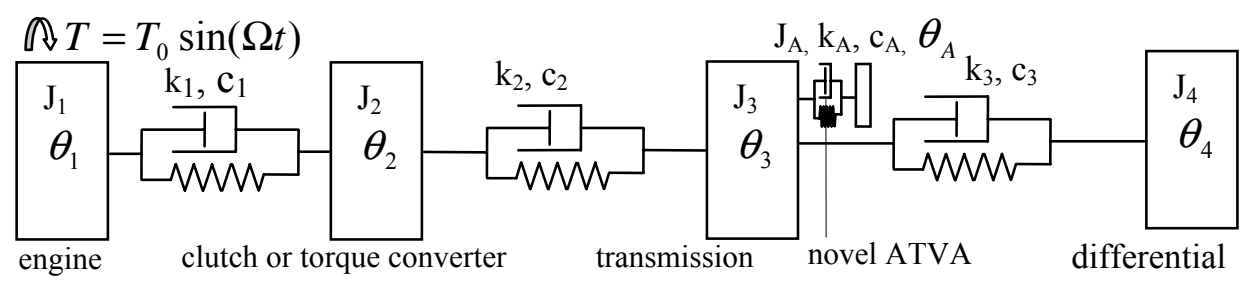

Fig 4. A simplified powertrain model with an ATVA

For such a model the Lagrange's equation is used to lead to the well-known equation of motion:

$$
J \ddot{\theta}+C \dot{\theta}+K \theta=T
$$

$\theta, \mathrm{T}$ are vectors of generalized coordinates and external torque. $\mathrm{J}, \mathrm{K}, \mathrm{C}$ are inertia, stiffness and damping matrices.

When an ATVA is attached, $\mathrm{J}_{\mathrm{A}}$ is fixed and the input current, $\mathrm{I}$, is tuned for controlling $\mathrm{k}_{\mathrm{A}}$. The conversion between $I$ and $\mathrm{k}_{\mathrm{A}}$ (also $\mathrm{f}_{\mathrm{A}}$ ) is through Eq.5 and Eq.6.

Generally, to solve Eq.7 for an arbitrary linear system with $\mathrm{N}$ degrees of freedom, Eq.7 is rewritten:

$$
\dot{z}=A z \text {. With } z=\left[\begin{array}{ll}
\theta & \dot{\theta}
\end{array}\right]^{T}, A=\left[\begin{array}{cc}
{[0]_{N \times N}} & {[E]_{N \times N}} \\
-J^{-1} K & -J^{-1} C
\end{array}\right], \text { E is the identity matrix. }
$$

Vibration features of Eq.7 such as natural frequencies, damped natural frequencies and damping ratios are determined from solving the eigenvalue problem of Eq. 8

$$
\lambda_{j}=h_{j} \pm i \omega_{d j}, \omega_{n j}=\sqrt{h_{j}^{2}+\omega_{d j}^{2}}, \varsigma_{j}={ }^{-h_{j}} / \omega_{n j} \text { for } \mathrm{j}=1 . . \mathrm{N} \text { and } i=\sqrt{-1} \text {. }
$$

For the steady motion, use complex form: $\theta=\Theta e^{i \Omega t}, T=T_{0} e^{i \Omega t}$. Eq. 7 will be $J \ddot{\theta}+C \dot{\theta}+K \theta=T_{0} e^{i \Omega t}$ and then the steady responses are determined by:

$$
\theta=\operatorname{Re}(\Theta) \cos (\Omega t)-\operatorname{Im}(\Theta) \sin (\Omega t) \text { with } \Theta=\left[-\Omega^{2} J+i \Omega C+K\right]^{-1} T_{0}
$$

\section{An application of ATVA for the second gear}

Let $\mathrm{J}_{1}=0.2, \mathrm{~J}_{2}=\mathrm{J}_{3}=0.1, \mathrm{~J}_{4}=3 \mathrm{kgm}^{2} ; \mathrm{c}_{1}=1.5, \mathrm{c}_{2}=\mathrm{c}_{3}=0.5 \mathrm{Nms} / \mathrm{rad} ; \mathrm{k}_{1}=2064, \mathrm{k}_{3}=484 \mathrm{Nm} / \mathrm{rad}$. Gear shifts modeled by coefficient $\mathrm{k}_{2}$ given in Table 1 .

Table 1. Natural frequency of simplified powertrain model for the second and third gear

\begin{tabular}{|c|cc|cc|}
\hline \multicolumn{2}{|c|}{ The second gear $\left(k_{2}=600[\mathrm{Nm} / \mathrm{rad}]\right)$} & \multicolumn{2}{c|}{ The third gear $\left(k_{2}=720[\mathrm{Nm} / \mathrm{rad}]\right)$} \\
\hline \multicolumn{2}{|c|}{ Natural frequency $[\mathrm{Hz}]$} & Damped frequency $[\mathrm{Hz}]$ & Natural frequency $[\mathrm{Hz}]$ & Damped frequency $[\mathrm{Hz}]$ \\
\hline $\mathrm{f}_{1}$ & 4.6802 & 4.6798 & 4.8231 & 4.8226 \\
$\mathrm{f}_{2}$ & 16.5631 & 16.5450 & 17.278 & 17.2610 \\
$\mathrm{f}_{3}$ & 30.3086 & 30.2316 & 30.8847 & 30.8089 \\
\hline
\end{tabular}

As shown in figure 4, the engine is subjected to a torque $T=T_{0} \sin (\Omega t)=10 \sin (2 \pi \times 4.68 t)$. Here
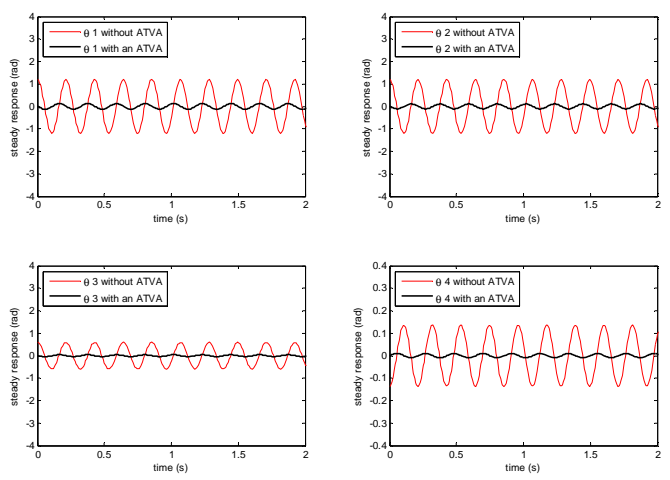

$\Omega=2 \pi \times 4.68 \mathrm{rad}$ coincides with $\omega_{n 1}=2 \pi \times f_{1}$ in Table 1 so that a novel ATVA whose natural frequency $\mathrm{f}_{\mathrm{A}}$ is attached. Due to $\mathrm{f}_{\mathrm{A}}=4.68 \mathrm{~Hz}$ the current $\mathrm{I}=1.215719 \mathrm{~A}$ is converted. Choose $\mathrm{I}=1.2 \mathrm{~A}, \mathrm{f}_{\mathrm{A}}=4.6 \mathrm{~Hz}$ is converted one more time, the powertrain vibration before and after adding the ATVA is shown in fig 5. Obviously, vibrations of all four disks, $\theta_{1}, \theta_{2}, \theta_{3}, \theta_{4}$ after adding ATVA (bold lines) are reduced significantly, that means the ATVA works well. Similar results is obtained for the second and third natural frequencies $\mathrm{f}_{2}=16.54$ and $\mathrm{f}_{3}=30.23 \mathrm{~Hz}$.

Fig 5. Steady responses at current $\mathrm{I}=1.2 \mathrm{~A}$

\section{An application of ATVA for the third gear}

Here $T=10 \sin (2 \pi \times 4.82 t)$. Let $\mathrm{f}_{\mathrm{A}}=4.82 \mathrm{~Hz}, \mathrm{I}=1.324514 \mathrm{~A}$ is converted; choose $\mathrm{I}=1.3 \mathrm{~A}\left(\mathrm{f}_{\mathrm{A}}=4.79 \mathrm{~Hz}\right)$ rather than $\mathrm{I}=1.2 \mathrm{~A}\left(\mathrm{f}_{\mathrm{A}}=4.68 \mathrm{~Hz}\right)$ for the second gear. The steady responses are displayed in fig $6 \mathrm{a}, \mathrm{b}$. 
In fig.6a the ATVA's effectiveness is limited because I=1.2A is the current for the second gear and it has been improved a lot when $\mathrm{I}=1.3 \mathrm{~A}$, in fig. $6 \mathrm{~b}$, due to $\mathrm{f}_{\mathrm{A}}$ is tuned to coincide with $\mathrm{f}_{1}=4.82 \mathrm{~Hz}$.
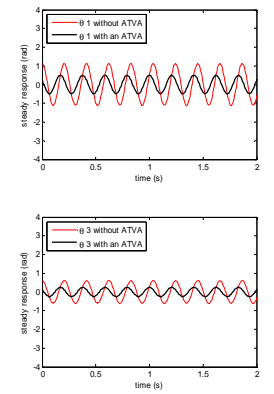

(a) $\mathrm{I}=1.2 \mathrm{~A}\left(\mathrm{f}_{\mathrm{A}}=4.66 \mathrm{~Hz}\right)$
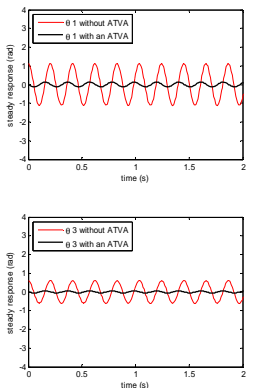

(b) $\mathrm{I}=1.3 \mathrm{~A}\left(\mathrm{f}_{\mathrm{A}}=4.79 \mathrm{~Hz}\right)$
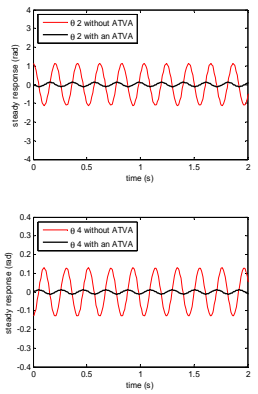

Fig 6. Steady responses of powertrain for third gear for two values of input current I

\section{Application of ATVA for second gear under a multi harmonic excitation}

In this case the powertrain subjected to a multi-harmonic excitation, in fig.7. Let $\mathrm{J}_{\mathrm{A} 1}=\mathrm{J}_{3} / 10$, $\mathrm{J}_{\mathrm{A} 2}=\mathrm{J}_{2} / 10, \mathrm{~T}_{10}=10, \mathrm{~T}_{20}=280 \mathrm{Nm}$, tune the input currents $\mathrm{I}_{1}=1.2, \mathrm{I}_{2}=13.7 \mathrm{~A}$ for ATVA1 and ATVA2.

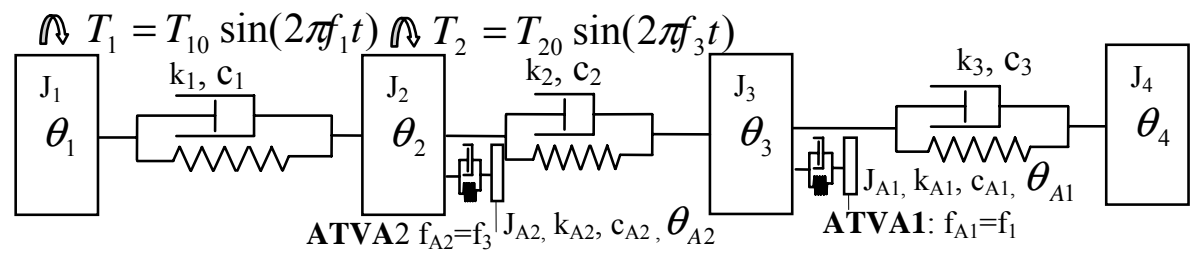

Fig 7. The powertrain with two ATVAs for the second gear

The steady responses are given in fig.8. Obviously, ATVAs worked well because the powertrain vibration is reduced greatly after these ATVAs attached (bold lines with respect to normal lines).
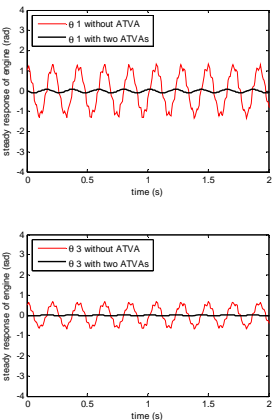
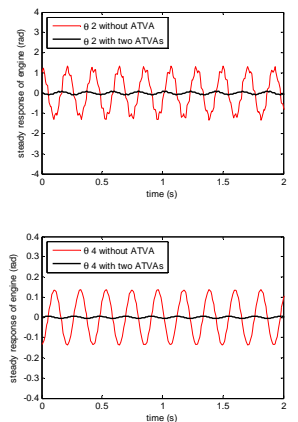

\section{Conclusions}

An enhanced MRE has been used to construct a novel ATVA and simulation results show that the ATVA's effectiveness for reducing powertrain vibration during gear shifts is significant. Also, the ATVA is effective for both cases of single harmonic excitation and multi-harmonic excitations. Moreover, the study proposed an MRE's squeeze-shear mode just in case the ATVA's frequency range is insufficient. This simulation will be useful for optimization of the ATVA's design in our next work.

Fig 8. Responses with two ATVAs

\section{Reference}

[1] A.R.Crowther: Transient vibration in Powertrain systems with Automatic Transmissions, Doctorate Thesis in University of Technology, Sydney, (2004).

[2] A.M. Albanese: The Design and Implementation of a Magnetorheological Silicone Composite State-Switched Absorber. Master. Sci, Georgia Institute of Technology, 2005.

[3] X.Zhang, X.Li, An effective permeability model to predict field-dependent modulus of magnetorheological elastomers, Commu Nonlinear Sci Numer Simul (2007), in press.

[4] M.Farshad, A.Benine: Magnetoactive elastomer composites, Polymer Testing 23(2004)347-353.

[5] G.V.Stepanov, S.S. Abramchuk, D.A. Grishin, L.V. Nikitin, el all: Effect of a homogeneous magnetic field on the viscoelastic behavior of magnetic elastomers, Polymer 48 (2007) 488-495.

[6] M.J. Garc1'a Ta'rrago, L.Kari, J.Vin olas el all: Torsion stiffness of a rubber bushing: a simple engineering design formula including the amplitude dependence, J. Strain Analysis 42, (2007)

[7] E.J.Park, D.Stoikov, L.Falcao, A.Suleman, a performance evaluation of an automotive magnetorheological brake design with a sliding mode controller, Mechatronics, 16,2006:405-416 